\title{
Video Article \\ Absorption of Nasal and Bronchial Fluids: Precision Sampling of the Human Respiratory Mucosa and Laboratory Processing of Samples
}

\author{
Ryan S Thwaites ${ }^{1}$, Hannah C Jarvis ${ }^{1}$, Nehmat Singh ${ }^{1}$, Akhilesh Jha ${ }^{1}$, Andy Pritchard ${ }^{2}$, Hailing Fan ${ }^{1}$, Tanushree Tunstall ${ }^{1}$, Joan Nanan ${ }^{1}$, \\ Simon Nadel $^{2}$, Onn Min Kon ${ }^{2}$, Peter J Openshaw ${ }^{1}$, Trevor T Hansel ${ }^{1}$ \\ ${ }^{1}$ National Heart and Lung Institute, Faculty of Medicine, Imperial College London, St Mary's Hospital \\ ${ }^{2}$ St Mary's Hospital, Imperial College Healthcare Trust
}

Correspondence to: Trevor T Hansel at t.hansel@imperial.ac.uk

URL: https://www.jove.com/video/56413

DOI: doi:10.3791/56413

Keywords: Medicine, Issue 131, Nasal absorption, bronchial absorption, Respiratory, Sampling, Airway, Mucosa, Biomarkers, Personalized medicine

Date Published: $1 / 21 / 2018$

Citation: Thwaites, R.S., Jarvis, H.C., Singh, N., Jha, A., Pritchard, A., Fan, H., Tunstall, T., Nanan, J., Nadel, S., Kon, O.M., Openshaw, P.J., Hansel, T.T. Absorption of Nasal and Bronchial Fluids: Precision Sampling of the Human Respiratory Mucosa and Laboratory Processing of Samples. J. Vis. Exp. (131), e56413, doi:10.3791/56413 (2018).

\section{Abstract}

The methods of nasal absorption (NA) and bronchial absorption (BA) use synthetic absorptive matrices (SAM) to absorb the mucosal lining fluid (MLF) of the human respiratory tract. NA is a non-invasive technique which absorbs fluid from the inferior turbinate, and causes minimal discomfort. NA has yielded reproducible results with the ability to frequently repeat sampling of the upper airway.

By comparison, alternative methods of sampling the respiratory mucosa, such as nasopharyngeal aspiration (NPA) and conventional swabbing, are more invasive and may result in greater data variability. Other methods have limitations, for instance, biopsies and bronchial procedures are invasive, sputum contains many dead and dying cells and requires liquefaction, exhaled breath condensate (EBC) contains water and saliva, and lavage samples are dilute and variable.

BA can be performed through the working channel of a bronchoscope in clinic. Sampling is well tolerated and can be conducted at multiple sites in the airway. BA results in MLF samples being less dilute than bronchoalveolar lavage (BAL) samples.

This article demonstrates the techniques of NA and BA, as well as the laboratory processing of the resulting samples, which can be tailored to the desired downstream biomarker being measured. These absorption techniques are useful alternatives to the conventional sampling techniques used in clinical respiratory research.

\section{Video Link}

The video component of this article can be found at https://www.jove.com/video/56413/

\section{Introduction}

Most respiratory diseases cause an inflammatory response, and there is an urgent need for sampling from the respiratory mucosal surface in allergic rhinitis, viral and fungal infections, tuberculosis, asthma, chronic obstructive pulmonary disease, pulmonary fibrosis and lung cancer ${ }^{1}$. Nasopharyngeal aspirate (NPA), nasal lavage, and bronchoalveolar lavage (BAL) are common techniques for sampling the upper and lower airway. However, these techniques present considerable problems including poor tolerability, dilution of inflammatory mediators, and the inability to frequently repeat sampling ${ }^{2}$. One alternative to NPA sampling is the use of swabs, either nylon flocked, cotton, or rayon ${ }^{3,4}$, but these also have limitations, since they cause discomfort and damage to the nasal epithelium, and in some cases irreversible binding of inflammatory mediators ${ }^{5}$. These techniques cannot generally be repeated serially over an hour, and alternative techniques may be more effective for detection of low abundance cytokines and chemokines ${ }^{5,6}$. Additionally, the user variability associated with these techniques may generate inconsistencies in data, resulting in the requirement of larger patient cohorts.

Alternatively, absorption techniques using both natural and synthetic sponges have been used to collect MLF from mucosal surfaces. Ophthalmic sponges composed of natural cellulose (e.g., Weck-cel) have been used to sample saliva, cervical, and vaginal secretions ${ }^{7}$. In addition, synthetic sponges made from polyvinyl alcohol (PVA) and hydroxylated polyvinyl acetate (HPVA) have been utilised ${ }^{8}$. Seven different absorptive materials have been compared for sampling oral fluid prior to measuring antibodies ${ }^{9}$, while polyurethane mini-sponges have been used to collect human tears $^{10}$.

Filter paper consisting of natural cellulose from the cotton plant has been widely used to absorb nasal secretions since the pioneering paper of Alam and colleagues in $1992^{11,12,13,14,15,16,17}$. Filter paper discs have been produced from filter cards (e.g. Shandon), and have been utilized to measure histamines and cytokines after controlled nasal allergen challenges and with natural allergen exposure ${ }^{18,19,20,21}$. However, different batches of filter paper vary in their degree of protein binding and some fail to release cytokines. Methods using a synthetic absorptive 
matrix (SAM) have therefore been developed ${ }^{2,22,23}$. SAMs are now generally used to obtain nasal MLF by NA. These absorbent materials are comfortable to use and can obtain MLF even from inflamed noses at frequent intervals over extended periods of time.

Nasal absorption is a form of Precision Mucosal Sampling using a SAM for the sampling of MLF in the upper airway. NA devices are manufactured as CE-marked medical devices from medical grade materials using clean rooms and are free of dust and allergens. The NA sampler consists of a handle and SAM in a sterile cryotube. The SAM consists of polymers, typically fibers, but it is also available as foam, and these are selected to be soft and absorptive, with rapid wicking for sample collection. SAMs have minimal protein binding to allow the efficient elution of absorbed secretions. NA is a very gentle, non-invasive technique that can be performed on donors of all ages. In addition, serial sampling, even every few minutes, is possible. NA has been validated against existing upper airway sampling techniques ${ }^{5}$ and repetitive sampling has allowed generation of kinetic data following challenge of the airway with allergen ${ }^{23,24,25}$, bacterial endotoxin ${ }^{26}$ and viral-type TLR $^{2}$ agonists (Jha, A. et al., manuscript in preparation). NA has also been used in infants to investigate the natural history of atopy ${ }^{27,28,29}$ and in viral bronchiolitis ${ }^{30}$.

Bronchoscopic microsampling (BMS) is a procedure for collection of MLF in the lower airway that has been developed by Olympus ${ }^{31,32,33}$. Unfortunately, this BMS system is only licensed in Japan. Olympus supply two BMS systems: one with a fibrous hydroxylated polyester (FHPE) probe $^{34,35,36,37}$, and one with a cotton probe ${ }^{33,38,39,40,41,42,43}$. A major stumbling block has been that the BMS probe used in patients with asthma caused mucosal contact bleeding, with half of all samples contaminated with blood. The authors concluded that it was not feasible to sample MLF using this BMS system from peripheral airways in asthma patients ${ }^{43}$.

As an alternative, we have developed BA using a soft SAM that can be performed during bronchoscopic investigation of the lower airways, including following experimental infection of asthmatic subjects with rhinovirus ${ }^{6}$. The BA device consists of: an external hollow catheter, a handpiece that on activation extrudes the SAM, and a central plastic guide wire that has the SAM on its end. As for NA, BA kits are manufactured from medical grade materials using clean rooms and are free of dust and allergens. Additionally, devices are CE marked and are provided gamma-irradiated. The SAM strip is soft, absorptive, and has rapid wicking for sample collection. It also has minimal protein binding to allow the efficient elution of absorbed secretions. The device can fit through the working channel of a bronchoscope and can be used to rapidly and accurately sample MLF at specific sites of interest within the airway. Unlike BAL or BMS, BA does not result in contact bleeding or additional patient discomfort post-procedure.

Careful consideration should be given to the processing of NA and BA samples. Samples can be directly frozen and processed in batches, or can be processed immediately. The type of processing can be tailored toward certain downstream applications, including immunoassays for cytokines, chemokines and immunoglobulins, or elutions of viral, bacterial, and host cell associated RNA. We present the clinical collection and laboratory processing techniques associated with NA and BA as a guide for clinical researchers.

\section{Protocol}

The techniques used in the following protocol have been approved by the West London Research Ethics Committee (Reference number 15/ 10/0444).

\section{Nasal Absorption (NA)}

\section{Preparation prior to NA sampling}

1. When carrying out NA, first wash hands and put on gloves, preferably in front of the patient.

2. Inspect the nasal cavity using a head torch, and have the clinician use their non-dominant thumb to retract the patient's nose to visualize the nasal cavity.

Note: A nasal speculum is not usually required.

3. Visualize the nasal cavity and inferior turbinate prior to sampling.

Note: The nares (nostrils) are not round in cross-section and they go straight backwards. Generally, the inferior turbinate can be seen as a bulge or indentation on the lateral wall of the nostril, with the nasal septum forming the smooth, flat medial wall. We want to sample from this inferior turbinate, since the underlying epithelium is a simple ciliated epithelium of the respiratory tract ${ }^{44}$.

\section{NA sampling}

1. When sampling, pass the NA SAM gently up the lumen of the nostril, orientating it to be flat against the inferior turbinate.

2. Ask the donor to use an index finger to press the SAM onto the nasal mucosa. NA can cause a slight tickling, with possible eye watering, as the MLF is absorbed.

Note: In adults, we generally perform NA for $60 \mathrm{~s}$.

3. After absorption of MLF, remove the NA device from the nostril and put back into the original tube.

4. Process the samples immediately in the laboratory or freeze them, as detailed later in this protocol.

Note: NA is being studied in a variety of nasal mucosal challenge models, and also in different airways diseases. However, validation against other respiratory sampling methods should be performed for each study and patient population.

\section{Bronchial Absorption (BA)}

\section{Preparation prior to $B A$ sampling}

Note: BA is performed by specialized personnel in a bronchoscopy suite.

1. Before placing the BA device down the catheter, check that the SAM is extruding and withdrawing back into the catheter.

2. Prior to conducting $B A$ on a patient, conduct a mock BA on a bronchoscopy simulator.

\section{BA sampling}


1. Pass the bronchoscope down the trachea and right main bronchus to the level of the bronchus intermedius, just proximal to the division into the right lower and right middle lobes.

NOTE: We typically sample from the bronchus intermedius, though other sites within the airway can be sampled. When observing the catheter and SAM, one cannot see the bronchoscope tip. We observe the following simple steps for BA, once the bronchoscope has reached the desired sampling site.

2. CATHETER DOWN: Insert the BA catheter through the operating channel of the bronchoscope until the white tip is just visible in the airway, to a maximum of $1 \mathrm{~cm}$ distal to the end of the bronchoscope. Keep the bronchoscope and catheter tip in the center of the lumen of the airway. Be careful to minimize contact between the catheter tip and the bronchial mucosa to reduce the risk of abrasion to the mucosa.

3. SAM OUT: Depress the handle of the BA device so that the SAM is extruded into the lumen of the right middle or right lower lobe airway. Under direct vision, bend the tip of the bronchoscope to ensure that the SAM is making contact with the MLF on the airway wall. Leave the SAM within the airway, flat to the mucosal wall for $30 \mathrm{~s}$.

4. SAM IN: Look through the bronchoscope to ensure that the moist SAM probe is straight and not bent back over the end of the catheter. If required, the catheter and bronchoscope tip can be brought back to straighten the SAM. Under direct vision, retract the straight SAM gently back into the catheter.

NOTE: If there is difficulty retracting the SAM back into the catheter, withdraw the whole device with SAM extruded back out of the airway.

5. CATHETER UP: Withdraw the entire catheter from the operating channel of the bronchoscope.

6. CUT OFF SAM: The SAM is cut off with sterile scissors and is then put into a cryotube on ice. These samples can be processed with varying methods, as detailed later in this protocol.

\section{Processing of NA and BA Samples}

Note: There are numerous options for laboratory processing of samples resulting from NA and BA. These protocols seek to store samples for later use, and to elute the MLF sample from the SAM.

\section{Options for immediate handling of NA and BA samples}

1. Option 1: Store the moist SAM on ice for a few hours prior to further processing.

2. Option 2: Immediately deep freeze NA SAMs in the collection tube. Similarly, freeze BA SAMs in cryogenic vials after removal, with scissors, from the sampling device.

3. Option 3: Place the detached SAM in elution buffer $(300 \mu \mathrm{L})$ prior to processing directly or deep freezing.

\section{Options of elution buffer for NA and BA samples}

Note: The choice of elution buffer depends on what is going to be analyzed in the MLF sample and we suggest four main alternatives:

1. Use a pre-prepared assay buffer suitable for immunoassay procedures. Such buffers should contain a small amount of detergent $(0.05 \%)$ as well as protein, e.g., bovine serum albumin (BSA) at $1 \%$.

2. Alternatively, use a buffer containing a greater amount of detergent, so that cell lysis occurs.

Note: We use buffers containing Triton-X or NP40 at $1 \%$ concentration. Cell lysis buffers enable both intracellular and extracellular cytokines to be eluted from the SAM, and generally result in higher levels of cytokines and chemokines. These buffers should also contain protein, and are made up with BSA to $1 \%$.

3. For RNA measurements, such as quantitative PCR of viral RNA or measuring host RNA, add RNA extraction buffer directly to the moist SAM.

Note: Chaotropic RNA extraction buffers contain guanidinium that denatures proteins. An alternative is to add RNA extraction buffer to the eluted MLF fluid contained in immunoassay or cell lysis buffer.

4. Use organic solvents, such as trifluoroacetic acid, for extraction of lipids and metabolites, for evaluation by mass-spectrometry. Note: Details of all these reagents are included in the Materials section.

\section{Elution technique}

1. For any of the above elution techniques, insert the SAM into a $2 \mathrm{~mL}$ micro-centrifuge tube, along with the desired extraction buffer.

2. Vortex mix the sample for $30 \mathrm{~s}$ to wash the SAM of loosely attached fluids and biomolecules.

3. To ensure full sample recovery, perform centrifugal elution by adding the moist SAM to a spin filter mini-column that inserts into the same $2 \mathrm{~mL}$ micro-centrifuge tube used for washing.

Note: Two types of spin filter mini-column can be used. The first contains only a plastic mesh, which holds the SAM in place, allowing full elution of fluids. Alternatively, if working with infectious materials, use spin filters with a $0.22 \mu \mathrm{m}$ pore size. These filters will sterilize samples and are suitable for samples with suspected Mycobacterial infection. However, these filters should be pre-incubated with buffer, to minimize binding of mediators to the filter by non-specific interactions.

4. Use sterilized forceps to transfer the moist SAM to the spin filter. Change forceps between samples to prevent contamination.

5. Centrifuge samples for $20 \mathrm{~min}$ at $16,000 \mathrm{xg}$ in a mini-centrifuge cooled to $4{ }^{\circ} \mathrm{C}$.

\section{Summary example protocol}

1. In the laboratory, label $2 \mathrm{~mL}$ micro-centrifuge tubes for sample collection and add the desired volume of elution buffer (typically $300 \mathrm{LL}$ for $\mathrm{NA} ; 100 \mu \mathrm{L}$ for BA). Seal lid and place on ice.

2. Following sampling (either immediately or in the lab) the SAM is removed from the handle using forceps and placed into the buffer containing collection tube (produced in previous step). Ensure the cap of the microcentrifuge tube is closed securely and transfer the samples, on ice, to the laboratory for further processing.

3. Remove tubes containing SAM and elution buffer from their transfer container and vortex mix for $30 \mathrm{~s}$.

4. Using sterile forceps, remove SAM and place into a spin column (with or without $0.22 \mu \mathrm{m}$ cellulose acetate filter).

5. Collect the elution buffer from the collection tube and retain. 
6. Place the spin column, with SAM, in the original collection tube (or a new one if sterile-filtering samples) and add collection buffer to spin column. In this manner, the wash fluid will pass through the spin column back into the collection tube, helping to elute the sample from the SAM.

7. Centrifuge the samples with lids sealed $\left(16,000 \times \mathrm{g}, 20 \mathrm{~min}, 4^{\circ} \mathrm{C}\right)$.

8. Remove the samples from the centrifuge and place into a rack.

9. Open the sealed lid of the tubes and remove the spin column containing the SAM. Dispose of SAM and spin column in an appropriate waste container.

10. Carefully aliquot the eluate contained within the tube into labelled cryogenic tubes. Record the total volume of eluate and the volume in each aliquot.

11. Transfer the sealed cryogenic tubes to $\mathrm{a}-80^{\circ} \mathrm{C}$ freezer and store upright until use.

\section{Representative Results}

NA has been utilized in a number of studies to easily and non-invasively measure mucosal inflammation. Following administration of allergen to the nose, prostaglandin-D2 (PGD2) levels can be observed to rise and fall within minutes (Figure 1), in line with mast cell degranulation (Thwaites et al., manuscript in preparation). Additionally, mediators of type-II inflammation, such as IL-5 (Figure 2, reprinted with permission from $\left.{ }^{24}\right)$, can be measured in the hours following nasal allergen challenge ${ }^{23,24}$. In experimental infection of allergic asthmatics and healthy controls, NA was used to measure a panel of mediators, including interferon-gamma (IFN- $y$ ), over the course of 7 days (Figure 3, reprinted with permission from ${ }^{6}$ ). Additionally, in natural respiratory syncytial virus (RSV) infection of infants, NA demonstrated RSV to be associated with elevated levels of inflammatory cytokines, such as IFN-y, relative to non-RSV infants with bronchiolitis and healthy controls (Figure 4, reprinted with permission from ${ }^{30}$ ). Interestingly, this discrimination between RSV and non-RSV bronchiolitis was not significant in time-matched NPA samples (Figure 4).

BA was also used during experimental infection of allergic asthmatics with rhinovirus. At day 4 of rhinovirus infection, levels of IFN- $\gamma, \mathrm{CXCL} 11$, IL-10, and IL-5 were elevated from baseline (Figure 5; A, B, C and D, respectively). Additionally, this technique demonstrated elevated IL-5 levels in the lower airway of allergic asthmatics during rhinovirus infection, relative to healthy controls (Figure 5D)(Figure 5 reprinted with permission from ${ }^{6}$ ).

These representative results were generated from samples eluted using assay buffer containing $0.05 \%$ Tween-20 and $1 \%$ BSA (see Table of Materials).

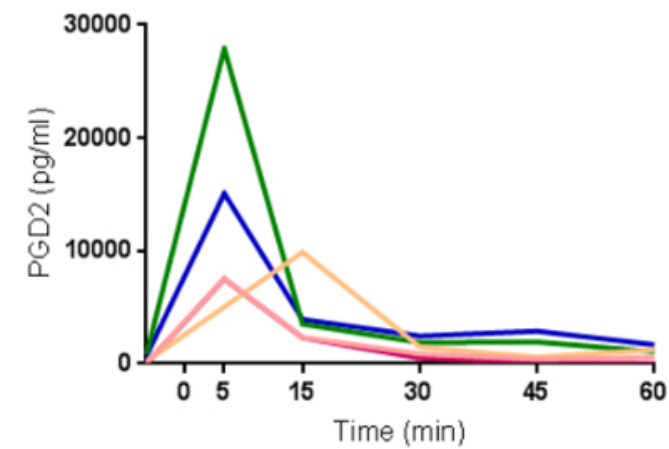

Figure 1: Rapid generation and clearance of prostaglandin-D2 following nasal allergen challenge. Levels of prostaglandin-D2 (PGD2) measured from nasal absorption eluates in serial samples following nasal allergen challenge with Timothy grass pollen ( $n=5)$. Each line represents data from one individual. Please click here to view a larger version of this figure. 


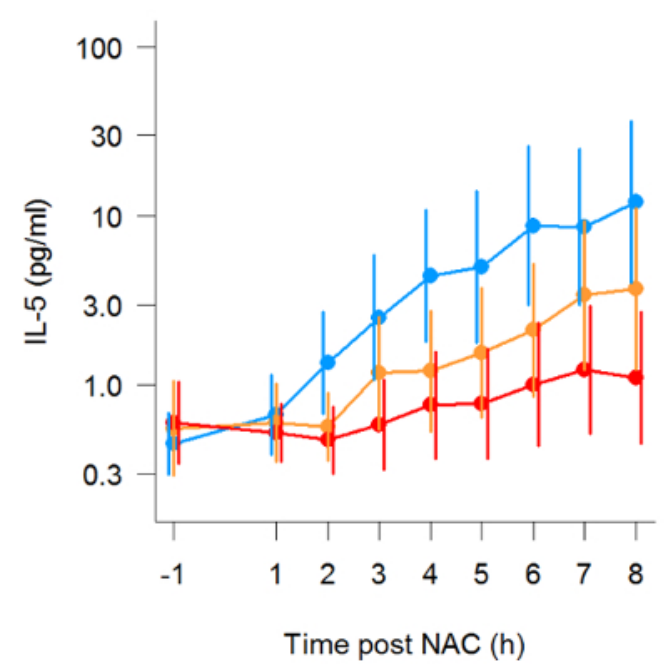

Figure 2: Kinetic measurement of IL-5 following nasal allergen challenge. Production of IL-5 in serial nasal absorption samples following nasal allergen challenge. Three repeat allergen challenge studies were conducted, with the participants $(n=19)$ receiving placebo (blue), low dose $(10 \mathrm{mg}$ ) oral prednisone (orange), or high dose $(25 \mathrm{mg}$ ) oral prednisone (red) one hour prior to allergen administration. Lines denote geometric mean and error bars are $95 \%$ confidence intervals of all participants. (Figure reprinted with permission from Leaker et al., Mucosal Immunology, 2017). Please click here to view a larger version of this figure.

A.

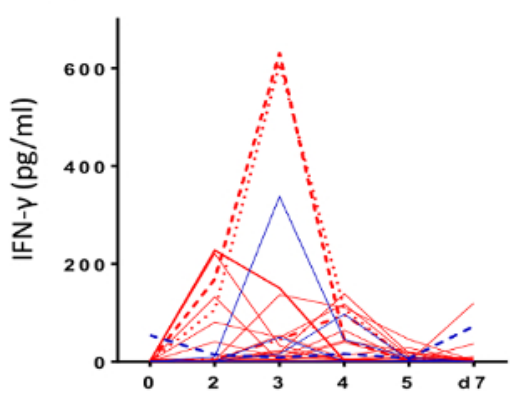

B.

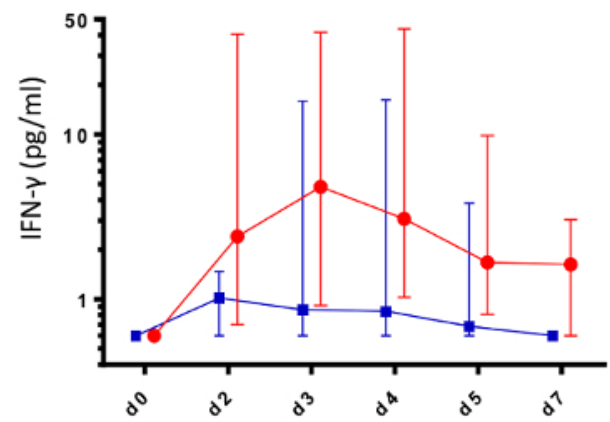

Figure 3: Induction of Interferon-y during rhinovirus infection. During infection challenge of healthy adults ( $n=11$, blue) and allergic asthmatics ( $n=28$, red) with rhinovirus-16, nasal absorption sampling was used to measure levels of interferon- $\mathrm{Y}$ (IFN- $\mathrm{\gamma}$ ). Data are represented as A) raw spaghetti plots of individuals and B) median levels with error bars denoting interquartile ranges. (Figure reprinted with permission from Hansel et al. ${ }^{6}$ ). Please click here to view a larger version of this figure.

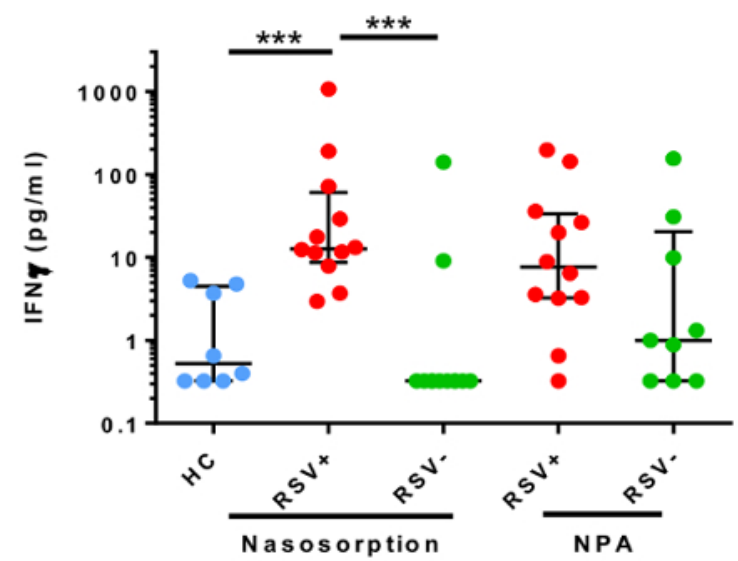

Figure 4: Nasosorption discriminates elevated Interferon-y associated with RSV infection of infants. Nasal absorption and nasopharyngeal aspiration (NPA) were used to measure interferon-y levels in infants with bronchiolitis associated with respiratory syncytial virus (RSV) infection (red, $n=12$ ), a non-RSV respiratory pathogen (green, $n=12$ ), and healthy controls (blue, $n=9$ ). Data were analyzed using a Kruskall-Wallis test with Dunns correction for multiple comparisons $\left({ }^{* * *} p<0.001\right)$. Lines denotes median values and error bars are interquartile ranges. (Figure modified with permission from Thwaites et al. ${ }^{30}$ ). Please click here to view a larger version of this figure. 

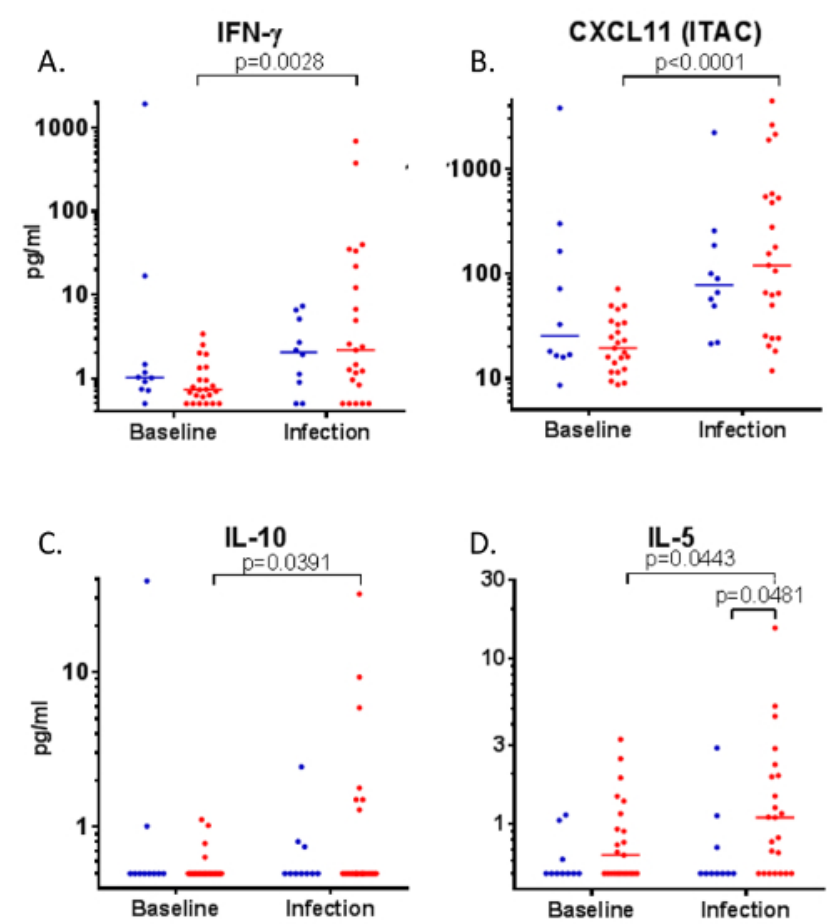

Figure 5: Inflammatory mediators in bronchial absorption samples during rhinovirus infection.

Following rhinovirus-16 infection of healthy controls $(n=10$, blue) and allergic asthmatic volunteers ( $n=23$, red), bronchial absorption was used to measure levels of A) IFN-y, B) CXCL11, C) IL-10, and D) IL-5 at baseline and on day 4 of infection. Data analyzed by Wilcoxon signed rank test (matched samples) and Mann-Whitney test (unmatched samples). Figure reprinted with permission from Hansel et al. ${ }^{6}$ Please click here to view a larger version of this figure.

\section{Discussion}

Results from existing airway sampling techniques are regarded as highly variable; alternative sampling techniques are needed to standardize research within this field ${ }^{5}$. NA and BA permit sampling of MLF in a non-invasive manner, and have exciting potential to measure immune responses in healthy and diseased airways. These techniques offer numerous potential advantages over existing techniques, including greater tolerability, speed of sampling, the ability to frequently repeat sampling, lower inter-user variability, and decreased dilution of immune mediators ${ }^{5}$. The duration of absorption and the processing technique used should be optimized for each study and rigorously maintained between sampling events. Additionally, in the case of BA, the site of sampling within the airway should be carefully replicated between individuals.

NA, and particularly BA, are still relatively novel techniques for clinical research. However, the benefits of these techniques have resulted in their use in numerous studies, including careful validation against alternative techniques ${ }^{5}$. These devices are now available as CE-marked devices ready for widespread use in respiratory research. While NA and BA result in much smaller sample volumes than alternative sampling techniques, higher obtained concentrations can result in greater sensitivity for low abundance immune mediators.

Depending on desired downstream applications, NA and BA samples can be directly frozen for later processing, enhancing study feasibility in a clinical research environment. The protocol for sample handling can also be adapted to suit particular downstream applications. The suggested processing techniques can be used for collection of protein or lipid immune mediators or nucleic acids, but should be optimized for each study. In particular, MLF can be eluted with different buffers. Firstly, immunoassay buffer can be used to measure mucosal cytokines, chemokines, and antibodies ${ }^{6,45}$. Buffers with higher detergent levels can also be used to guarantee cell lysis occurs, allowing the inclusion of intra-cellular cytokines. Chaotropic RNA extraction buffers should be used for the determination of viral infection, viral load, host mRNA, and the microbiome. Alternatively, organic solvents can be used for lipidomics and mass spectrometry.

In conclusion, direct absorption of MLF from mucosal surfaces is an exciting technique with potential use in respiratory, gastro-intestinal, urogenital, and other mucosal diseases. However, these promising absorption techniques will require precise validation of sampling and processing technique for individual assays (biomarkers) in each disease setting. In addition, these novel precision mucosal sampling techniques will require validation against conventional samples, such as from blood, breath, and sputum. With these techniques, MLF can be used to measure microbes, cytokines, chemokines, prostanoids, and antibodies.

\section{Disclosures}

The authors have nothing to disclose. 


\section{Acknowledgements}

Funding: This work was supported by funding from the Imperial National Institute for Health Research (NIHR) Biomedical Research Centre (BRC), the NIHR Health Protection Research Unit (HPRU) in Respiratory Infections at Imperial College London in partnership with Public Health England (PHE) and the NIHR Imperial Patient Safety Translational Research Centre. The views expressed are those of the authors and not necessarily those of the NHS, the NIHR, the Department of Health or Public Health England.

\section{References}

1. Hansel, T. T., Johnston, S. L., \& Openshaw, P. J. Microbes and mucosal immune responses in asthma. Lancet. 381 (9869), 861-873 (2013).

2. Lu, F. X., \& Esch, R. E. Novel nasal secretion collection method for the analysis of allergen specific antibodies and inflammatory biomarkers $J$ Immunol Methods. 356 (1-2), 6-17 (2010).

3. Esposito, S. et al. Collection by trained pediatricians or parents of mid-turbinate nasal flocked swabs for the detection of influenza viruses in childhood. Virol J. 7 (1), 85 (2010).

4. Macfarlane, P., Denham, J., Assous, J., \& Hughes, C. RSV testing in bronchiolitis: which nasal sampling method is best? Arch Dis Child. 90 (6), 634-635 (2005).

5. Jochems, S. P. et al. Novel Analysis of Immune Cells from Nasal Microbiopsy Demonstrates Reliable, Reproducible Data for Immune Populations, and Superior Cytokine Detection Compared to Nasal Wash. PLoS One. 12 (1), e0169805 (2017).

6. Hansel, T. T. et al. A Comprehensive Evaluation of Nasal and Bronchial Cytokines and Chemokines Following Experimental Rhinovirus Infection in Allergic Asthma: Increased Interferons (IFN-gamma and IFN-lambda) and Type 2 Inflammation (IL-5 and IL-13). EBioMedicine. (2017).

7. Rohan, L. C. et al. Optimization of the weck-Cel collection method for quantitation of cytokines in mucosal secretions. Clin Diagn Lab Immunol. 7 (1), 45-48 (2000).

8. Castle, P. E. et al. Comparison of ophthalmic sponges for measurements of immune markers from cervical secretions. Clin Diagn Lab Immunol. 11 (2), 399-405 (2004).

9. Chang, C. K., Cohen, M. E., \& Bienek, D. R. Efficiency of oral fluid collection devices in extracting antibodies. Oral Microbiol Immunol. 24 (3), 231-235 (2009).

10. Lopez-Cisternas, J., Castillo-Diaz, J., Traipe-Castro, L., \& Lopez-Solis, R. O. Use of polyurethane minisponges to collect human tear fluid. Cornea. 25 (3), 312-318 (2006).

11. Alam, R., Sim, T. C., Hilsmeier, K., \& Grant, J. A. Development of a new technique for recovery of cytokines from inflammatory sites in situ. $J$ Immunol Methods. 155 (1), 25-29 (1992).

12. Sim, T. C., Grant, J. A., Hilsmeier, K. A., Fukuda, Y., \& Alam, R. Proinflammatory cytokines in nasal secretions of allergic subjects after antigen challenge. Am J Respir Crit Care Med. 149 (2 Pt 1), 339-344 (1994).

13. Sim, T. C., Reece, L. M., Hilsmeier, K. A., Grant, J. A., \& Alam, R. Secretion of chemokines and other cytokines in allergen-induced nasal responses: inhibition by topical steroid treatment. Am J Respir Crit Care Med. 152 (3), 927-933 (1995).

14. Weido, A. J., Reece, L. M., Alam, R., Cook, C. K., \& Sim, T. C. Intranasal fluticasone propionate inhibits recovery of chemokines and other cytokines in nasal secretions in allergen-induced rhinitis. Ann Allergy Asthma Immunol. 77 (5), 407-415 (1996).

15. Linden, M. et al. Immediate effect of topical budesonide on allergen challenge-induced nasal mucosal fluid levels of granulocyte-macrophage colony-stimulating factor and interleukin-5. Am J Respir Crit Care Med. 162 (5), 1705-1708 (2000).

16. Bensch, G. W., Nelson, H. S., \& Borish, L. C. Evaluation of cytokines in nasal secretions after nasal antigen challenge: lack of influence of antihistamines. Ann Allergy Asthma Immunol. 88 (5), 457-462 (2002).

17. Riechelmann, H., Deutschle, T., Friemel, E., Gross, H. J., \& Bachem, M. Biological markers in nasal secretions. Eur Respir J. 21 (4), 600-605 (2003).

18. Wagenmann, M. et al. Bilateral increases in histamine after unilateral nasal allergen challenge. Am J Respir Crit Care Med. 155 (2), $426-431$ (1997).

19. Wagenmann, M., Schumacher, L., \& Bachert, C. The time course of the bilateral release of cytokines and mediators after unilateral nasal allergen challenge. Allergy. 60 (9), 1132-1138 (2005).

20. Baumann, R. et al. The release of IL-31 and IL-13 after nasal allergen challenge and their relation to nasal symptoms. Clin Transl Allergy. 2 (1), 13 (2012).

21. Baumann, R. et al. Nasal levels of soluble IL-33R ST2 and IL-16 in allergic rhinitis: inverse correlation trends with disease severity. Clin Exp Allergy. 43 (10), 1134-1143 (2013).

22. Chawes, B. L. et al. A novel method for assessing unchallenged levels of mediators in nasal epithelial lining fluid. J Allergy Clin Immunol. 125 (6), 1387-1389 e1383 (2010).

23. Scadding, G. W. et al. Optimisation of grass pollen nasal allergen challenge for assessment of clinical and immunological outcomes. $J$ Immunol Methods. 384 (1-2), 25-32 (2012).

24. Leaker, B. R. et al. The nasal mucosal late allergic reaction to grass pollen involves type 2 inflammation (IL-5 and IL-13), the inflammasome (IL-1beta), and complement. Mucosal Immunol. 10 (2), 408-420 (2017).

25. Nicholson, G. C. et al. The effects of an anti-IL-13 mAb on cytokine levels and nasal symptoms following nasal allergen challenge. $J$ Allergy Clin Immunol. 128 (4), 800-807 e809 (2011).

26. Dhariwal, J. et al. Nasal Lipopolysaccharide Challenge and Cytokine Measurement Reflects Innate Mucosal Immune Responsiveness. PLoS One. 10 (9), e0135363 (2015).

27. Folsgaard, N. V. et al. Neonatal cytokine profile in the airway mucosal lining fluid is skewed by maternal atopy. Am J Respir Crit Care Med. 185 (3), 275-280 (2012).

28. Folsgaard, N. V. et al. Pathogenic bacteria colonizing the airways in asymptomatic neonates stimulates topical inflammatory mediator release. Am J Respir Crit Care Med. 187 (6), 589-595 (2013).

29. Wolsk, H. M. et al. Siblings Promote a Type 1/Type 17-oriented immune response in the airways of asymptomatic neonates. Allergy. 71 (6), 820-828 (2016). 
30. Thwaites, R. S. et al. Nasosorption as a Minimally Invasive Sampling Procedure: Mucosal Viral Load and Inflammation in Primary RSV Bronchiolitis. J Infect Dis. 215 (8), 1240-1244 (2017).

31. Ishizaka, A. et al. New bronchoscopic microsample probe to measure the biochemical constituents in epithelial lining fluid of patients with acute respiratory distress syndrome. Crit Care Med. 29 (4), 896-898 (2001).

32. Ishizaka, A. et al. Elevation of KL-6, a lung epithelial cell marker, in plasma and epithelial lining fluid in acute respiratory distress syndrome. Am J Physiol Lung Cell Mol Physiol. 286 (6), L1088-1094 (2004).

33. Komaki, Y. et al. Cytokine-mediated xanthine oxidase upregulation in chronic obstructive pulmonary disease's airways. Pulm Pharmacol Ther. 18 (4), 297-302 (2005).

34. Yamazaki, K., Ogura, S., Ishizaka, A., Oh-hara, T., \& Nishimura, M. Bronchoscopic microsampling method for measuring drug concentration in epithelial lining fluid. Am J Respir Crit Care Med. 168 (11), 1304-1307 (2003).

35. Kikuchi, J., Yamazaki, K., Kikuchi, E., Ishizaka, A., \& Nishimura, M. Pharmacokinetics of telithromycin using bronchoscopic microsampling after single and multiple oral doses. Pulm Pharmacol Ther. 20 (5), 549-555 (2007).

36. Kikuchi, E. et al. Comparison of the pharmacodynamics of biapenem in bronchial epithelial lining fluid in healthy volunteers given half-hour and three-hour intravenous infusions. Antimicrob Agents Chemother. 53 (7), 2799-2803 (2009).

37. Kodama, T. et al. A technological advance comparing epithelial lining fluid from different regions of the lung in smokers. Respir Med. 103 (1), 35-40 (2009).

38. Sasabayashi, M., Yamazaki, Y., Tsushima, K., Hatayama, O., \& Okabe, T. Usefulness of bronchoscopic microsampling to detect the pathogenic bacteria of respiratory infection. Chest. 131 (2), 474-479 (2007).

39. Kipnis, E. et al. Proteomic analysis of undiluted lung epithelial lining fluid. Chest. 134 (2), 338-345 (2008).

40. Kanazawa, H., Kodama, T., Asai, K., Matsumura, S., \& Hirata, K. Increased levels of N(epsilon)-(carboxymethyl)lysine in epithelial lining fluid from peripheral airways in patients with chronic obstructive pulmonary disease: a pilot study. Clin Sci (Lond). 119 (3), 143-149 (2010).

41. Sugasawa, Y. et al. The effect of one-lung ventilation upon pulmonary inflammatory responses during lung resection. $J$ Anesth. 25 (2), 170-177 (2011).

42. Sugasawa, Y. et al. Effects of sevoflurane and propofol on pulmonary inflammatory responses during lung resection. $J$ Anesth. 26 (1), $62-69$ (2012).

43. Cohen, J. et al. Ciclesonide improves measures of small airway involvement in asthma. Eur Respir J. 31 (6), 1213-1220 (2008).

44. Fahy, J. V., \& Dickey, B. F. Airway mucus function and dysfunction. N Engl J Med. 363 (23), 2233-2247 (2010).

45. de Silva, T. I. et al. Comparison of mucosal lining fluid sampling methods and influenza-specific IgA detection assays for use in human studies of influenza immunity. J Immunol Methods. (2017). 